\title{
Alimentación de mamíferos de caza en los «aguajales» de la Reserva Nacional de Pacaya-Samiria (Iquitos, Perú)
}

\author{
Feeding of game animals on «aguajales» in the Reserva Nacional Pacaya \\ Samiria (lquitos, Peru)
}

\author{
Rolando Aquino*
}

Presentado: $05 / 01 / 2005$

Aceptado: $\quad 19 / 09 / 2005$

\section{Resumen}

El presente trabajo contiene información relacionada a los mamíferos de caza que habitan los aguajales de los bosques inundables de la Reserva Nacional Pacaya Samiria y su interrelación con las plantas alimenticias. Está basado en censos por transectos en aguajales de origen reciente y aguajales semi-eutrofizados. Fueron registrados 24 especies de mamíferos de caza haciendo uso de los aguajales; de ellas, los primates fueron los más representativos y de mayor abundancia en ambos tipos de aguajales. Entre los primates, el fraile (Saimiri boliviensis E. Geoffroy \& R. de Blainville) y el machín negro (Cebus apella Linnaeus) fueron los más abundantes en aguajales de origen reciente (350 individuos $/ \mathrm{km}^{2}$ y 90 individuos $/ \mathrm{km}^{2}$, respectivamente), mientras que el machín blanco (Cebus albifrons Humboldt) fue el más abundante en aguajales semi-eutrofizados (90 individos/ $\mathrm{km}^{2}$ ). En términos de biomasa, los ungulados tuvieron la mayor biomasa y estuvieron representados por el pecarí labiado (Tayassu pecari Link) con $825 \mathrm{~kg} / \mathrm{km}^{2}$ y el tapir (Tapirus terrestris Linnaeus) con $126,6 \mathrm{~kg} / \mathrm{km}^{2}$. Finalmente, en los aguajales fueron registradas 16 especies de plantas alimenticias, la mayoría habitando en los aguajales semi-eutrofizados; de ellas, el aguaje (Mauritia flexuosa L.f.), la shapaja (Scheelea cephalotes Poepp. Ex Mart) y los renacos (Ficus spp.), constituyeron los recursos alimenticios más importantes, de cuyos frutos y semillas se alimentaron alrededor de 14 especies de mamíferos de caza.

Palabras clave: Pacaya Samiria, aguajales, Mauritia flexuosa, mamíferos de caza, Reservas Naturales, conservacion.

\begin{abstract}
This report contains information related to the game mammals that inhabit the palm swamps (aguajales) of the flooded forests of the Reserva Nacional Pacaya Samiria and their interrelation with the food plants. It is based on censuses by transects in aguajales of recent origin and semi-eutrophic aguajales. 24 species of game mammals were registered making uses of the aguajales; of them, the primates were the most representative and the great abundance in both aguajales types. Among the primates, the Squirrel monkey (Saimiri boliviensis E. Geoffroy \& R. de Blainville) and the brown capuchin monkey (Cebus apella Linnaeus) were the most abundant in aguajales of recent origin (350 individuals $/ \mathrm{km}^{2}$ and 90 individuals $/ \mathrm{km}^{2}$, respectively), while the white-fronted capuchin monkey (Cebus albifrons Humboldt) it was the most abundant in aguajal semi-eutrofizado ( 90 individuals $/ \mathrm{km}^{2}$ ). In terms of biomass, the ungulates had the biggest biomass and they were represented by the white-lipped peccary (Tayassu pecari Link) with $825 \mathrm{~kg} / \mathrm{km}^{2}$ and the tapir (Tapirus terrestris Linnaeus) with 126,6 kg/ $/ \mathrm{km}^{2}$. Finally, 16 species of food plants were registered in aguajales, most of them inhabiting semi-eutrophic aguajales; of those the aguaje (Mauritia flexuosa L.f.), the shapaja (Scheelea cephalotes Poepp. Ex Mart) and the renacos (Ficus spp.) constituted the most important food resources of whose fruits and seeds fed around 14 species of game mammals.
\end{abstract}

Key words: Pacaya Samiria, aguajales, palm swamps, Mauritia flexuosa, game mammals, Nature Reserves, conservation

* Instituto de Ciencias Biológicas Antonio Raimondi, Facultad de Ciencias Biológicas, Universidad Nacional Mayor de San Marcos. Apartado 11-0058, Lima 11, Perú. Estación Experimental del IVITA-Iquitos, Perú. Telefax 65-265510.

Email Rolando Aquino: ivitaiq@terra.com.pe 


\section{Introducción}

Los típicos aguajales se desarrollan en los bosques inundables y constituyen un tipo de bosque dominado por las palmeras de aguaje (Mauritia flexuosa L.f. ), asociadas al aguajillo (Mauritiella sp.), al huasaí (Euterpe oleraceae Mart.) y otras especies con formas de vida especializadas y adaptadas a los hábitats hidrometamórficos de estuarios y várzeas, así como los terrenos pantanosos de tierra firme.

Estos bosques casi monotípicos, tienen una alta producción de frutos y son importantes recursos alimenticios para un grupo de especies, al menos así lo indican los análisis de contenidos estomacales de ciertas especies de mamíferos de caza (Bodmer, 1990; Bodmer et al., 1997; Bodmer et al., 1999) y los resultados obtenidos en estaciones de muestreo (Allen, 1997). Los frutos de aguaje también constituyen alimento importante para la gente ribereña y para los que viven en el cinturón de las grandes ciudades de la Amazonia, quienes lo consumen en bebidas, y helados (Padoch, 1987; Vásquez y Gentry, 1989). Por otra parte, este tipo de bosques también constituye el hábitat para una gran variedad de insectos, arácnidos, batracios y lacértidos, por lo tanto contribuyen indirectamente en la alimentación de los mamíferos arborícolas, particularmente forrajeros como los machines (Cebus spp.), pichicos (Saguinus spp.) y frailes (Saimiri spp.). Además, los troncos secos sirven de madrigueras para un grupo importante de mamíferos arborícolas de hábitos nocturnos (Aquino y Encarnación, 1986) y para la nidificación de algunas especies de aves, particularmente psitácidos como los guacamayos y loros.

Los aguajales se encuentran amenazados por la demanda de sus frutos. La cosecha de los frutos de aguaje mediante técnicas inadecuadas como el derribamiento del tronco es cada vez mayor y esto tiene un impacto en las cercanías a las comunidades ribereñas, probablemente con un mayor efecto en las especies de caza más pequeñas, como el añuje (Dasyprocta fuliginosa Wagler), majaz (Agouti paca Linnaeus) y las ratas espinosas (Proechimys spp.). Si la demanda continúa a mayor escala en las grandes ciudades y no se difunden técnicas más sustentables de cosecha, como la del uso de subidores, desarrollada en la comunidad de Parinari en el río Marañón, es predecible que el derribamiento se extenderá a lugares más lejanos del bosque e impactarán a las especies más grandes de la fauna silvestre, originando una paulatina disminución tanto de aguajales como de la fauna asociada a él.

Los aguajales con terreno fangoso y pantanoso son lugares de cacería de los moradores ribereños en período de «creciente» (febreromayo) por el fácil acceso mediante el uso de canoas, lo que no ocurre en período de «vaciante» (junio - diciembre). Esta dinámica explica en parte la carencia de estudios en este tipo de bosques sobre todo los referidos a la interrelación planta-animal, también motivó a la conducción del presente estudio y para cuyo efecto nos propusimos los siguientes objetivos: a) inventariar los mamíferos de caza que habitan y/o hacen uso de los aguajales, b) estimar la densidad poblacional y biomasa de los mamíferos de caza y c) determinar las especies de plantas que son utilizadas en la alimentación por estos animales.

\section{Áreas de estudio}

Los estudios fueron llevados a cabo en la Reserva Nacional Pacaya Samiria de agosto a setiembre de 1998, octubre a noviembre del 2000, setiembre a octubre del 2001, setiembre a noviembre del 2002 y enero del 2003, y comprendieron los aguajales aledaños al curso medio de la quebrada Yanayacu de Pucate, cuyas aguas son vertidas directamente al río Marañón cerca de la ciudad de Nauta; San Miguel, próximo a la confluencia entre los ríos Samiria y Marañón y Parinari en la quebrada de Parinari. También comprendieron los aguajales de Tacsha Cocha en el curso medio del río Samiria y quebradas de Guanaico, Armana, y Pinche, afluentes de la quebrada Yanayacu grande (Fig. 1) De acuerdo a la última zonificación (INRENA, 2000), estos 
aguajales correspondieron a la zona de uso directo y de recuperación de la Reserva. En San Miguel, Tacsha Cocha y Pinche, los censos por transecto con fines de inventario y para estimar densidad poblacional fueron conducidos en aguajales de origen reciente; en Yanayacu de Pucate, Guanaico y Armana en aguajales semieutrofizados y en Parinari, en aguajales de origen reciente y semi-eutrofizados. Con excepción de los aguajales de Tacsha Cocha, Guanaico, Armana y Pinche, los demás presentaron signos de haber sufrido desde moderada a una fuerte alteración, resultado de la cosecha de frutos de aguaje (M.flexuosa) y de la extracción de palmito del huasaí (E. oleraceae), ambos por derribamiento del tronco. La caza en estos aguajales aparentemente fue moderada, probablemente por el difícil acceso en época de «vaciante».

\section{Material y métodos}

Los componentes del estudio fueron los mamíferos de caza que hacen uso de este tipo de bosques y las plantas alimenticias que habitan los aguajales y/o forman parte de su hábitat.

Para los censos fue utilizado el método de transecto lineal. Con esta finalidad, en cada uno de los aguajales tuvimos que abrir entre 2 a 3 trochas de 1 a 3,0 $\mathrm{km}$ de longitud. Los censos fueron conducidos por las mañanas desde las 06:00 a 11:00 h y por las tardes desde las 14:00 a 17:00 h (hora local). Los censos consistieron en el registro de grupos y/o individuos de mamíferos mediante visualización directa. Cada vez que hubo contacto procedimos a anotar la especie, número de individuos, y la distancia perpendicular animal-transecto.

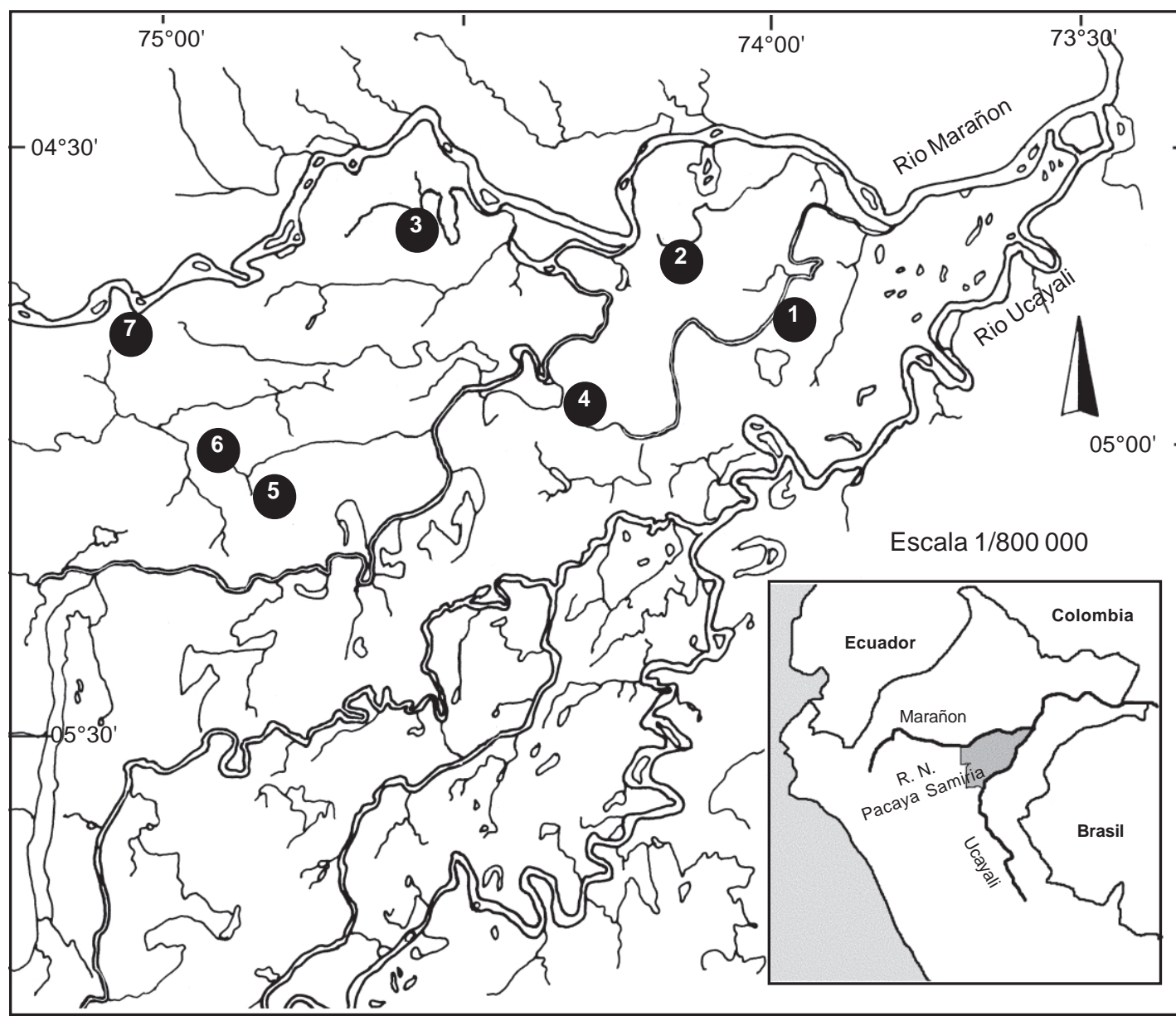

Figura 1. Mapa de la Reserva Nacional Pacaya Samiria mostrando las áreas de estudio: 1) quebrada Yanayacu de Pucate, 2) San Miguel, 3) quebrada Parinari, 4) Tacsha Cocha, 5) quebrada Guanaico, 6) quebrada Armana y 7) quebrada Pinche. 
Tabla 1. Áreas censadas y longitudes recorridas en aguajales de la Reserva Nacional Pacaya Samiria.

\begin{tabular}{llr}
\hline Áreas censadas & Tipo de aguajal & $\begin{array}{r}\text { Longitud } \\
\text { Recorrida } \\
(\mathbf{k m})\end{array}$ \\
\hline Yanayacu de & Semi-eutrofizado & 59 \\
Pucate & Origen reciente & 100 \\
San Miguel & Semi-eutrofizado & 93 \\
Parinari & Origen reciente & 30 \\
Tacsha Cocha & Semi-eutrofizado & 16 \\
Guanaico & Semi-eutrofizado & 36 \\
Armana & Origen reciente & 11 \\
Pinche & & 345 \\
Total: & & \\
\hline
\end{tabular}

Para el caso de animales de hábitos nocturnos, la verificación de su presencia en los aguajales fue por hostigamiento en sus madrigueras que generalmente fueron huecos en troncos secos de aguaje tanto en pié como en los caídos. Por otro lado, cuando los animales eran sorprendidos alimentándose de frutos y/o semillas, éstos fueron registrados simultáneamente en los censos, procediendo a la colecta de restos de frutos y/o semillas para su posterior identificación previo tratamiento con una mezcla de alcohol y formol, rotulado y almacenado en bolsas plásticas. Adicionalmente, hicimos caminatas nocturnas en tramos cortos en aguajales semi-eutrofizados con la finalidad de registrar a las especies de hábitos nocturnos más no así para la estimación de densidad poblacional. En total fueron censados $141 \mathrm{~km}$ en aguajales de origen reciente y $204 \mathrm{~km}$ en aguajales semieutrofizados (Tabla 1).

El reducido número de registros para la mayoría de las especies en cada uno de los aguajales fue insuficiente para la estimación de densidades mediante el uso del método DISTANCE que requiere un mínimo de 25 muestras por transecto (Buckland et al., 1993); por lo que se utilizó el método de transecto lineal (Brockelman y Alí, 1987), cuya fórmula es $\mathrm{D}=\mathrm{N} / 2 \mathrm{WL}$, donde $\mathrm{D}$ es la densidad, $\mathrm{N}$ es el número de los animales avistados, $\mathrm{W}$ es el promedio del ancho y L es la longitud de la trocha censada. Para el caso de especies que viven en grupos, el número de individuos $/ \mathrm{km}^{2}$ fue calculado multiplicando el número de grupos por el tamaño promedio.

\section{Resultados}

\section{Mamíferos registrados en aguajales}

Un total de 24 especies de mamíferos, entre arborícolas y terrestres fueron registradas haciendo uso de los aguajales. Entre los arborícolas que fueron la mayoría, hubieron 10 especies de primates habitando ambos tipos de aguajales, entre los que se encontraron el maquizapa de frente amarilla (Ateles belzebuth E. Geoffroy) y el maquizapa negro (Ateles chamek Linnaeus), ambas ausentes en las restingas y llanuras de la Reserva en mención. Entre los terrestres que fueron registrados únicamente en aguajales semieutrofizados no hubo predominio y los escasos contactos correspondieron a tres especies de roedores, dos de ungulados y cuatro de carnívoros (Tabla 2). El piso algo compacto de estos aguajales podría haber influido en cierta medida para el acceso de especies terrestres, incluso de los animales considerados de tamaño grande como el tapir (Tapirus terrestris Linnaeus) y el pecarí labiado (Tayassu pecari Link).

\section{Densidad poblacional y biomasa}

La densidad y biomasa de mamíferos de caza fueron estimadas únicamente para las especies de hábito diurno. Los resultados indicaron mayor densidad y biomasa de mamíferos de caza en aguajales semi-eutrofizados (Tabla 3). Los primates fueron los más abundantes en ambos tipos de aguajales; no obstante, a nivel de biomasa el pecarí labiado fue el más representativo con $825,0 \mathrm{~kg} / \mathrm{km}^{2}$. Entre los primates, con excepción del machín blanco (Cebus albifrons Humbolt), las densidades más altas para el fraile (Saimiri boliviensis E. Geoffroy \& R. de Blainville) (280 individuos $/ \mathrm{km}^{2}$ ), machín negro (Cebus apella Linnaeus) ( 90 individuos $/ \mathrm{km}^{2}$ ) y pichico pardo (Saguinus fuscicollis Spix) (22,8 indi- 
viduos $/ \mathrm{km}^{2}$ ), fueron registradas en aguajales de origen reciente, lo que indicaría que este tipo de bosque constituye un óptimo hábitat para estos primates, particularmente para el machín negro y el fraile. En cuanto al machín blanco, la mayor densidad fue registrada en aguajal semi-eutrofizado (54,6 individuos $\left./ \mathrm{km}^{2}\right)$.

\section{Plantas alimenticias y uso por los animales}

En los aguajales, además del aguaje ( $M$. flexuosa), huasaí (E. oleraceae), aguajillo (Mauritiella sp.) y shapaja (Scheelea cephalotes Poepp. Ex Mart) que fueron las que predominaron, también registramos otras 12 especies de plantas alimenticias habitando este tipo de bosques, la mayoría de ellas en los

Tabla 2. Mamíferos de caza registrados en aguajales del bosque inundable de la Reserva Nacional Pacaya Samiria

\begin{tabular}{|c|c|c|c|}
\hline \multirow[t]{2}{*}{ Especies } & \multirow[t]{2}{*}{ N ombre común } & \multicolumn{2}{|c|}{ Tipo de aguajal } \\
\hline & & $\begin{array}{l}\text { O rigen } \\
\text { reciente }\end{array}$ & $\begin{array}{c}\text { Semi- } \\
\text { eutrofizado }\end{array}$ \\
\hline \multicolumn{4}{|l|}{ O rden Perissodactyla: } \\
\hline 1. Tapirus ter restris & Tapir & $A$ & $\mathrm{P}$ \\
\hline \multicolumn{4}{|l|}{ O rden A rtiodactyla: } \\
\hline 2. Tayassu pecari & Pecarí labiado & $A$ & $\mathrm{P}$ \\
\hline \multicolumn{4}{|l|}{ O rden Rodentia: } \\
\hline 3. Proechimys sp. ${ }^{1}$ & Sachacuy & $A$ & $P$ \\
\hline 4. Sciurus spadiceus & Ardilla colorada & $A$ & $\mathrm{P}$ \\
\hline 5. D asyprocta fuliginosa & Añuje & $A$ & $\mathrm{P}$ \\
\hline 6. A gouti paca ${ }^{1}$ & Majáz & $A$ & $\mathrm{P}$ \\
\hline \multicolumn{4}{|l|}{ Orden Carnívora: } \\
\hline 7. Panthera onca & Otorongo & $A$ & $\mathrm{P}$ \\
\hline 8. Eira barbara & Manco & $A$ & $\mathrm{P}$ \\
\hline 9. $\mathrm{N}$ asua nasua & Achuni & $A$ & $\mathrm{P}$ \\
\hline 10. Potos flavus ${ }^{1}$ & Chosna & $\mathrm{P}$ & $\mathrm{P}$ \\
\hline 11. A telocynus microtis & Perro de monte & $A$ & $\mathrm{P}$ \\
\hline 12. Procyon cancrivorus ${ }^{1}$ & Perro conchero & $A$ & $\mathrm{P}$ \\
\hline \multicolumn{4}{|l|}{ O rden Primates: } \\
\hline 13. A teles belzebuth & Maquizapa frente amarilla & $P$ & $\mathrm{P}$ \\
\hline 14. A teles chamek & Maquizapa negro & $P$ & $P$ \\
\hline 15. Lagothrix poeppigii & Choro & $\mathrm{P}$ & $\mathrm{P}$ \\
\hline 16. A louatta seniculus & Mono aullador & $\mathrm{P}$ & $\mathrm{P}$ \\
\hline 17. Cebus apella & Machín negro & $P$ & $P$ \\
\hline 18. Cebus albifrons & Machín blanco & $P$ & $P$ \\
\hline 19. Pihecia monachus & Huapo negro & $\mathrm{P}$ & $P$ \\
\hline 20. Saimir boliviensis & Fraile & $P$ & $P$ \\
\hline 21. A otus nancymae ${ }^{1}$ & Musmuqui & $P$ & $\mathrm{P}$ \\
\hline 22. Saguinus fuscicollis & Pichico pardo & $\mathrm{P}$ & $P$ \\
\hline \multicolumn{4}{|l|}{ O rden Xenarthra: } \\
\hline 23. Tamandua tetradáctyla & Shihui & $A$ & $\mathrm{P}$ \\
\hline 24. Bradypus variegatus & Pelejo & $A$ & $P$ \\
\hline
\end{tabular}

${ }^{1}$ Mamíferos de hábitos nocturnos; $\mathrm{P}=$ Presente, $\mathrm{A}=$ A usente 
Tabla 3. Densidad y biomasa de mamíferos de caza estimadas para dos tipos de aguajales de la Reserva Nacional Pacaya Samiria.

\begin{tabular}{|c|c|c|c|c|}
\hline \multirow[t]{2}{*}{ Especies } & \multicolumn{2}{|c|}{$\begin{array}{l}\text { Aguajal origen } \\
\text { reciente }\end{array}$} & \multicolumn{2}{|c|}{$\begin{array}{c}\text { A guajal semi- } \\
\text { eutrofizado }\end{array}$} \\
\hline & $\begin{array}{l}\text { Densidad } \\
(\text { Ind.//km²) }\end{array}$ & $\begin{array}{l}\text { Biomasa } \\
\left(\mathrm{Kg} / \mathbf{k m}^{2}\right)\end{array}$ & $\begin{array}{l}\text { Densidad } \\
\text { (Ind./km²) }\end{array}$ & $\begin{array}{r}\text { Biomasa } \\
\left(\mathrm{Kg} / \mathrm{km}^{2}\right)\end{array}$ \\
\hline \multicolumn{5}{|l|}{ Orden Perissodactyla: } \\
\hline 1. Tapirus terrestris & 0,0 & 0,0 & 0,76 & 126,6 \\
\hline \multicolumn{5}{|l|}{ Orden Artiodactyla: } \\
\hline 2. Tayassu pecari & 0,0 & 0,0 & 25,0 & 825,0 \\
\hline \multicolumn{5}{|l|}{ Orden Rodentia: } \\
\hline 3. Sciurus spadiceus & 3,8 & 3,0 & 6,6 & 5,3 \\
\hline 4. D asyprocta fuliginosa & 0,0 & 0,0 & 1,8 & 9,0 \\
\hline \multicolumn{5}{|l|}{ Orden Carnívora: } \\
\hline 5. Panthera onca & 0,0 & 0,0 & 0,15 & 9,0 \\
\hline \multicolumn{5}{|l|}{ Orden Primates: } \\
\hline 6. A teles belzebuth & 2,0 & 22,0 & 4,2 & 46,0 \\
\hline 7. A teles chamek & 1,0 & 11,0 & 0,0 & 0,0 \\
\hline 8. Lagothrix poeppigii & 6,7 & 53,6 & 20,0 & 160,0 \\
\hline 9. A louatta seniculus & 11,5 & 92,0 & 11,2 & 89,6 \\
\hline 10. Cebus apella & 90,0 & 315,0 & 48,6 & 170,0 \\
\hline 11. Cebus albifrons & 16,8 & 50,4 & 54,6 & 163,8 \\
\hline 12. Pithecia monachus & 6,7 & 13,4 & 12,4 & 24,8 \\
\hline 13. Saimiri boliviensis & 350,0 & 280,0 & 336,0 & 268,8 \\
\hline 14. Saguinus fuscicollis & 22,8 & 11,4 & 3,6 & 1,8 \\
\hline
\end{tabular}

aguajales semi-eutrofizados (Tabla 4). Por otro lado, fue notoria la diferencia en cuanto a composición florística entre los aguajales de origen reciente y aguajales semieutrofizados. Los primeros se caracterizaron por ser un bosque relativamente abierto y poblado casi monotípicamente por árboles de aguaje y huasaí, matizado ralamente por otras cuatro especies de plantas alimenticias. En los aguajales semi-eutrofizados, el bosque presentó un aspecto más cerrado, donde además del aguaje, destacaron por su predominancia el huasaí, shapaja, ubus (Spondias mombin L.), catahua (Hura crepitans L.), ayahuma (Couroupita subsessilis Pilger) y cumala (Virola $\mathrm{sp}$.), entre otras (Tabla 3 ).

Entre las plantas alimenticias registradas en los aguajales, el aguaje (M. flexuosa) fue el más importante para los animales; en efecto, en 31 oportunidades fueron observados un to- tal de 10 especies de mamíferos alimentándose de sus frutos. Además del aguaje, también destacaron por su mayor frecuencia de consumo la shapaja y los renacos (Ficus spp.), en cuyos árboles los animales fueron igualmente sorprendidos comiendo de sus frutos en 26 y 18 oportunidades, respectivamente (Tabla 4 ). Aparte, los aguajales también contribuyeron indirectamente en la alimentación de los mamíferos, de cuyos bosques probablemente obtienen la mayor cantidad de proteína animal, particularmente los denominados forrajeros como los machines, pichicos y frailes. En efecto, en los aguajales de origen reciente, poblados casi exclusivamente por palmeras, las brácteas de las hojas constituyeron ambientes muy propicios para el desarrollo y escondite de muchos invertebrados y pequeños vertebrados. En estos bosques, los llamados forrajeros fueron frecuentemente encontrados en plena actividad de forrajeo; es decir, abriendo las brácteas en pro- 
Tabla 4. Lista de plantas que habitan en aguajales de la Reserva Nacional Pacaya Samiria, y que fueron siendo consumidas por mamíferos de caza. Se indica tipo de aguajal en que se encuentran, las veces en que fue observado el consumo y las especies que lo consumian.

\begin{tabular}{llcrr}
\hline Especies & \multicolumn{2}{c}{ Tipo de aguajal } & & \\
\cline { 2 - 3 } & $\begin{array}{c}\text { Origen } \\
\text { reciente }\end{array}$ & $\begin{array}{c}\text { Semi- } \\
\text { eutrofizado }\end{array}$ & $\begin{array}{c}\text { Frecuencia } \\
\text { de consumo }\end{array}$ & $\begin{array}{r}\text { M amíferos } \\
\text { consumidores }\end{array}$ \\
\hline
\end{tabular}

\section{Familia Annonaceae:}

1. A nnona duckei?

Familia Arecaceae:

2. Bactris sp.

3. Euterpe oleraceae

4. Geonoma sp.

5. Socratea sp.

6. Iriartea sp.

7. M auritia flexuosa

8. M auritiella peruviana? +

9. Scheel ea cephal otes

10. Scheelea sp.

\section{Familia Lecythidaceae:}

11. Couroupita subsessilis

Familia M yristicaceae:

12. Iryanthera sp.

13. Virola surinamensis

14. Virola pavonis?

\section{Familia M oraceae:}

15. Ficus spp.

16. Ficus antihelmintica

\begin{tabular}{|c|c|c|c|}
\hline - & + & 2 & $\mathrm{Sf}, \mathrm{Sb}$ \\
\hline+ & + & 3 & Cal, Sf \\
\hline+ & + & 4 & $\mathrm{Pm}$ \\
\hline+ & - & 1 & Cal \\
\hline- & + & 3 & Lp, Ca, As \\
\hline - & + & 4 & $A b$ \\
\hline+ & + & 31 & $\begin{array}{r}\text { Ab, Ach, Cal, Ca, Df, Ag, As, } \\
\text { Lp, Pm, Tp }\end{array}$ \\
\hline+ & + & 3 & $\mathrm{Ca}, \mathrm{Cal}, \mathrm{Pm}$ \\
\hline- & + & 26 & Lp, Ca, Cal, Se, Df, \\
\hline - & + & 2 & $\mathrm{Ca}, \mathrm{Se}$ \\
\hline - & + & 6 & Lp, Ca, Cal \\
\hline- & + & 1 & $\mathrm{Pm}$ \\
\hline- & + & 5 & $A b, L p, P m, S f$ \\
\hline- & + & 2 & $\mathrm{Pm}, \mathrm{Sf}$ \\
\hline+ & + & 18 & Lp, As, Sb, Sf \\
\hline- & + & 4 & As \\
\hline
\end{tabular}

$A b=A$. belzebuth, $A g=A$. paca, $A c h=A$. chamek, $A s=A$. seniculus, $C a=C$. apella, $C a l=C$. al bifrons, $D f=$ $D$. fuliginosa, $L p=L$. poeppigii, $\mathrm{Pm}=\mathrm{P}$. monachus, $\mathrm{Sb}=\mathrm{S}$. boliviensis, $\mathrm{Se}=\mathrm{S}$. espadiceus, $\mathrm{Sf}=\mathrm{S}$. fuscicollis, $\mathrm{Tp}=\mathrm{T}$. pecari.

cura de pequeños anfibios, insectos, arácnidos, lacértidos y huevos y polluelos de aves para complementar su dieta alimenticia.

La producción de frutos de aguaje en lo que corresponde a las áreas de estudio y probablemente para la cuenca del río Marañòn tuvo una periodicidad más ó menos prolongada y ocurrió entre noviembre y abril. La producción fue relativamente baja en los aguajales cercanos a los caseríos debido al reducido número de árboles hembras en pié por la indiscriminada tala para la cosecha de los frutos y fue relativamente alta en lugares como Tacsha Cocha, Guanaico, Armana y Parinari, los mismos que se encuentran bastante alejadas de los centros poblados. En estos aguajales fue común encontrar abundantes frutos al pié de los troncos durante el período de mayor fructificación y maduración (noviembre - enero). Precisamente en estos aguajales fueron observados comiendo los frutos de $M$. flexuosa a 7 especies de primates, un ungulado y dos roedores.

\section{Discusión}

Los resultados obtenidos nos indican claramente que la riqueza faunística varía entre uno y otro tipo de aguajales. Los aguajales de origen reciente se caracterizaron por presentar 
la más baja diversidad en cuanto a mamíferos, y estuvo constituida únicamente por las especies arborícolas. Lo contrario ocurrió en los aguajales semi-eutrofizados, cuya diversidad de animales fue casi similar a las obtenidas en las restingas y llanuras de la Reserva Nacional Pacaya Samiria (Bodmer et al., 1997, 2000) y cuenca del río Samiria (Aquino et al., 2001). Esta diferencia tiene mucha relación con el piso del bosque; que en el caso de los aguajales de origen reciente permanecieron totalmente cubiertos por masas de agua negra durante todo el año sin opción alguna incluso para el acceso de animales considerados como excelentes nadadores como el tapir y los pecaríes labiados. En los aguajales semi-eutrofizados, el acceso de animales terrestres tuvo mucho que ver con el piso que presentó un aspecto algo compacto y al menos cuatro meses del año permaneció relativamente seco (agosto-noviembre); además, las abundantes raíces zancas de los árboles hidro-metamórficos sirvieron para el acceso y como ambientes propicios para el descanso e incluso para el sueño de los animales

Entre los mamíferos que hicieron mayor uso de los aguajales figuran los primates y entre los ungulados el pecarí labiado y el tapir). El venado colorado (Mazama americana Erxleben) y el pecarí de collar (Pecari tajacu Linnaeus), dos asiduos visitantes de los aguajales de altura estuvieron ausentes, lo que demostraría una vez más que ambas especies están mejor adaptadas a los bosques de altura, mientras que en los bosques inundables su hábitat al parecer está limitado a las restingas altas (Bodmer et al., 2000).

La densidad y biomasa estimada para mamíferos de caza en aguajales semi-eutrofizados fueron igualmente mayores a las obtenidas por Bodmer et al. (1997, 2000) y Aquino et al. (2001) para los bosques inundables de la cuenca del río Samiria. Esta diferencia posiblemente tiene relación con la alta producción de frutos y la moderada presión de caza, cuyo piso fangoso y presencia de plantas espinosas parecerían determinantes para el acceso limitado de cazadores, siendo los más favorecidos las poblaciones de maquizapas (Ateles spp.) quienes encontraron en estos aguajales el refugio adecuado para protegerse de su principal depredador, el hombre. Sin embargo, es oportuno señalar que los censos para estimar densidades y biomasa fueron ejecutados durante la época de «vaciante», es decir, entre agosto y enero, coincidente con la escasez de frutos en los bosques de restingas y várzea, por lo que sería recomendable ejecutar los censos en época de «creciente», afín de comparar ambos resultados. Por el momento se asume que los aguajales estarían siendo visitados con mayor frecuencia en época de «vaciante», al menos así ocurrió en los aguajales de altura (Aquino, obs. pers.), donde los animales, particularmente primates solían ingresar a estos bosques poco después del amanecer y abandonaban al atardecer solo para pernoctar en sus respectivos árboles de dormir en los bosques de colina y terrazas.

En referencia a las plantas alimenticias, el aguaje probablemente es el recurso alimenticio más importante para los mamíferos en los bosques inundables de la Reserva Nacional Pacaya Samiria. Al respecto, los registros nos indican que los frutos de esta especie en comparación a las demás fueron proporcionalmente la más consumida y por una mayor diversidad de mamíferos. Los registros también indicaron como una de las pocas plantas con prolongada disponibilidad y abundancia de frutos en este tipo de bosques. Resultados similares fueron obtenidos por Aquino y Bodmer (en prensa) para primates en bosques inundables de la cuenca del río Samiria, quienes además sostienen que en este tipo de bosques el mayor número de especies de plantas que aportan en la alimentación están agrupados dentro de la familia Arecaceae.

\section{Conclusiones}

En la Reserva Nacional Pacaya Samiria, los aguajales cumplen un rol muy importante para un grupo de animales silvestres, el registro de 24 especies de mamíferos de caza así lo demuestran. 
En la Reserva Nacional Pacaya Samiria, los aguajales constituyen el único refugio para las escasas poblaciones de maquizapas ( $A$. belzebuth y A. chamek) que habitan en la citada reserva.

En los aguajales, los primates se caracterizaron por ser los más abundantes; sin embargo, los ungulados fueron los más representativos en biomasa.

La densidad y biomasa estimada para mamíferos de caza en aguajales fueron mayores a las estimadas para los bosques de várzea y restingas de la cuenca del río Samiria.

En los aguajales, el aguaje (M. flexuosa), shapaja (S. cephalotes) y los renacos (Ficus spp.) se constituyeron en los principales recursos alimenticios para la comunidad de mamíferos frugívoros.

\section{Agradecimientos}

Mi reconocimiento a Junglevagt For Amazonas AIF-WWF/DK, Programa Integral de Desarrollo y Conservación Pacaya Samiria que financió el estudio: «Evaluación de la fauna silvestre en San Miguel y Parinari con miras al manejo sostenible con participación comunitaria». Al Consejo Superior de Investigaciones (CSI) de la Universidad Nacional Mayor de San Marcos, por el financiamiento del estudio: «El rol de los aguajales en la alimentación de los animales silvestres de caza». Al Instituto Nacional de Recursos Naturales (INRENA) y a la Jefatura del Pacaya Samiria por facilitarnos el permiso para el ingreso a la mencionada reserva. Mi gratitud a los guías de campo de las comunidades de Yarina, asentada en la quebrada Yanayacu de Pucate, Parinari, asentada en la quebrada del mismo nombre y Nueva esperanza en el Marañón, por su activa participación durante la apertura de trochas en los aguajales.

\section{Literatura citada}

Allen, C. 1997. Frugivores: Palms and Conservation in Amazonia. M. Sc. Thesis, University of Florida, Gainesville.

Aquino, R. y F. Encarnación. 1986. Characteristics and use of sleeping sites in Aotus (Cebidae: Primates) in the Amazon lowland of Peru. Am. J. of Primatol. 11: 319 - 331.

Aquino, R.; R. E. Bodmer, y G. Gil. 2001. Mamíferos de la Cuenca del río Samiria: Ecología poblacional y sustentabilidad de la caza. Imprenta Rosegraf. Lima, Perú.

Bodmer, R.;R. Aquino y P. Puertas. 1997. Alternativas de manejo para la Reserva Nacional Pacaya Samiria: Un análisis sobre el uso sostenible de la caza. En: T. Fang, R. Bodmer, R. Aquino y M. Valqui (eds): Manejo de Fauna Silvestre en la Amazonia. La Paz, Bolivia: 65 - 74.

Bodmer, R E. y R. Aquino. 2000. Ecología poblacional de mamíferos en bosques inundables de la Amazonia Peruana. En: E. Cabrera, C. Mercolli y R. Resquin (eds): Manejo de Fauna Silvestre en Amazonia y Latinoamérica. Asunción, Paraguay: $153-165$.

Bodmer, R. E. 1990. Responses to ungulates to seasonal inundation in the Amazon floodplain. J. of Trop. Ecol. 6: 191 - 201.

Bodmer, R.E.; C. Allen; J. Penn; R. Aquino y C. Reyes. 1999. Evaluación del uso sostenible de la fauna silvestre en la Reserva Nacional Pacaya Samiria. Documento de Trabajo América Verde No. 4b. The Nature Conservancy.Arlington, USA.

Brockelman, R. y R. Alí 1987. Methods of surveying and sampling forest primate population. En: C. W. Marash y R. A. Mittermeier (ed): Primate Conservation in Tropical Rain Forest. New York: $23-63$

Buckland, S.T.; D. P. Anderson; K. P. Burnham y J. L. Laake. 1993. Distance sampling estimating abundance of biological populations. Chapman \& Hall, London. U.K.

INRENA, 2000. Plan Maestro de la Reserva Nacional Pacaya Samiria. Iquitos, Perú.

Padoch, C. 1987. The economic importance and marketing of forest and fallow products in the Iquitos region. Adv. in Econ. Bot. 5: 74 - 89.

Vásquez, R. y A. H. Gentry. 1989.Use and misuse of forest-harvested fruits in the Iquitos area. Conservation Biology 3: 350 - 361. 